\title{
Análisis de datos de la obtención de ácido láctico a partir de residuos de piña (Ananas comosus)
}

\section{Data Analysis of Obtaining Lactic Acid from Pineapple Residues (Ananas comosus)}

Recibido: 20 de noviembre de 2020. Recibido en revisión: 04 de diciembre de 2020. Aceptado: 17 de marzo de 2021. DOl: https://doi.org/10.33132/27114260.1905 Margie Alejandra Ripe Gutiérrez'; Eileen Xiomara Guerra', Martha Cervantes Díaz'

\section{Resumen}

El aprovechamiento de los residuos biodegradables provenientes del procesamiento de frutas y verduras representa una gran oportunidad de disminuir el impacto ambiental. Es el caso de los residuos de piña que sirven de materia prima para la obtención de diversos compuestos incluyendo ácido láctico, insumo de gran importancia en diferentes industrias. Esta investigación tuvo como objetivo analizar las tendencias de la publicación de artículos relacionados con la obtención de ácido láctico a partir del uso de piña y sus residuos como sustrato de partida. Se utilizaron 87 artículos obtenidos mediante ecuación de búsqueda en la base de datos Scopus (Elsevier, B.V., 2021) publicados entre 2010 y 2020. Aunque no se observa una tendencia definida en la distribución de los documentos por año, se percibe un gran interés en el tema; 2020 fue el año de mayor actividad científica (15 publicaciones); estos trabajos aplican, predominantemente, en diferentes campos de conocimiento - investigativo, inmunología y microbiología, seguidos de ciencia de materiales y en una menor proporción agricultura y ciencias biológicas- Los países con más publicaciones son Malasia y Tailandia con 15 documentos. En cuanto a los microorganismos más estudiados para la obtención de ácido láctico mediante proceso biotecnológico se identificaron el Lactobacillus plantarum y Escherichia coli. El aumento en el número de publicaciones refleja interés en la búsqueda de alternativas ambientales para la

1 Facultad de Química Ambiental, Programa de Maestría en Ciencias y Tecnologías Ambientales, Grupo de Investigaciones para el Desarrollo Sostenible, Universidad Santo Tomás, Bucaramanga. martha.cervantes(Dustabuca.edu.co, https://orcid.org/00000002-4427-687 margie.ripe@ustabuca.edu.co. 
valorización de estos residuos agroindustriales; obtención de compuestos de interés y aplicación en las áreas farmacéutica, cosmética e industrial, entre otras.

Palabras clave: ácido láctico, Ananas comosus, biotecnología, Lactobacillus, piña.

\section{Abstract}

The use of biodegradable waste from the processing of fruits and vegetables represents an excellent opportunity to reduce the environmental impact; for instance, pineapple residues serve as raw material for obtaining different compounds, including lactic acid, an input of great importance to different industries. This research discusses the trends in publishing articles related to obtaining lactic acid from pineapple and its residues as a starting substrate. We used 87 articles published between 2010 and 2020, obtained from a search equation in the Scopus (Elsevier, BV, 2021) database. Although there is no definite trend in the distribution of documents per year, there is great interest in the subject. The year 2020 featured the highest scientific activity (15 publications). These articles encompass various knowledge domains, especially immunology and microbiology, followed by materials science and, to a lesser extent, agriculture and biological sciences. The countries with the most publications are Malaysia and Thailand, with 15 papers. As for the most studied microorganisms to get lactic acid through a biotechnological process, Lactobacillus plantarum and Escherichia coli were identified. The increased number of publications shows an interest in searching for environmental alternatives to recover these agro-industrial residues and obtain compounds of interest for pharmaceutical, cosmetic, and industrial applications.

Keywords: Ananas comosus, pineapple, biotech, Lactobacillus, lactic acid. 


\section{Introducción}

La generación de residuos agroindustriales en el mundo representa un desafío ambiental, ya que al no ser aprovechados causan un deterioro progresivo del medio ambiente. Los desechos agroindustriales son residuos orgánicos sólidos que se producen durante la cosecha de frutas y la preparación de alimentos para consumo humano y animal; estos incluyen partes no comestibles de las frutas como cáscaras y semillas (Kuan y Liong, 2008). La creciente preocupación por el aprovechamiento de estos residuos ha producido interés en la comunidad científica debido a que pueden utilizarse como materia prima en la industria. Según (Beltrán-Ramírez et al., 2019) estos residuos agroindustriales brindan un enorme potencial para generar biomateriales, biocompuestos activos y bioenergía.

La mayoría de estos residuos terminan en vertederos o rellenos sanitarios provocando impacto ambiental negativo y deficiencia en la cadena de producción. El Área Metropolitana de Bucaramanga produce cerca de 350 Ton/semana de residuos sólidos en las plazas de mercado de los municipios pertenecientes a la misma; de la cantidad total, casi 220
Ton/semanales $(61,9 \%)$ son residuos de origen orgánico como cáscaras de piña, maracuyá y naranja que son depositados en el relleno sanitario El Carrasco. Una caracterización del tipo de desecho que se produce en la plaza de mercado de San Francisco mostró una generación de residuos cercana a 7 Ton/semana donde $25,1 \%$ corresponde a cáscaras de piña, seguida de cáscaras de cítricos $(9,2 \%)$ (Vargas A., 2016).

Los residuos sólidos de piña se consideran materia prima de diferentes productos. Son fuente de fibra, contienen abundante celulosa, hemicelulosa y otros carbohidratos, enzimas (e.g., bromelina), azúcares (e.g., fructosa) y polifenoles, (e.g., el ácido ferúlico) (Aida et al., 2011) (Ketnawa et al., 2012).

Otro compuesto que se obtiene a partir de la cáscara de la piña es el ácido láctico - LA, por sus siglas en ingléscomo se observa en la figura 1. Industrialmente puede ser producido por síntesis química o producción biotecnológica. La principal desventaja de la síntesis química es la obtención de mezclas racémicas de ácido D (-) y L (+) láctico, debido a que la separación de los isómeros es compleja y costosa. La obtención por producción biotecnológica se lleva a cabo con microorganismos cuya principal ventaja 


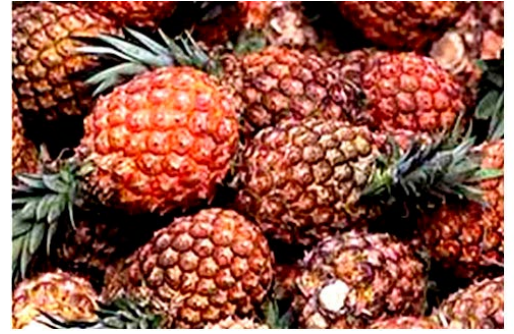

a)

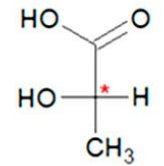

Isómero $(+)$

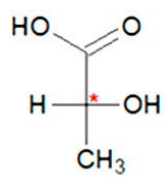

Isómero (-)

b)

Figura 1. Estructura de los isómeros del ácido láctico.

Nota. Isómeros del ácido láctico que pueden obtenerse a partir de la piña Ananas comosus. a) Adaptado de Ananas sp. [Fotografía], por Salcedo, 2016, Institucional de Documentación Científica Instituto de Investigación de Recursos Biológicos Alexander von Humboldt.

(http://repository.humboldt.org.co/handle/20.500.11761/1520)b) Licencia: ACD/ChemScketh Freeware versión 2020.1.1

Fuente: Autor, 2021.

es la alta especificidad del producto ya que produce el estereoisómero deseado, L (+) o D (-) LA ópticamente puro. (Torre, 2019).

La importancia de esta investigación radica en la estrategia que permite disminuir la cantidad de residuos agroindustriales no aprovechados; evitar vertimientos - al no ser dispuestos directamente en rellenos sanitarios-; también busca valorizar el residuo a través de la obtención de LA mediante producción biotecnológica; esto es decisivo, debido a que se emplean fuentes renovables que no tienen en cuenta síntesis químicas producen, finalmente, ácido láctico puro de potencial uso industrial.
El LA tiene gran aplicación en la industria alimentaria, como saborizante, conservante o acidulante. Se ha demostrado que posee una alta capacidad para inhibir el crecimiento de patógenos que podrían alterar las características organolépticas de los alimentos. En el caso de la industria cosmética se usa como humectante, regulador de $\mathrm{pH}$ y electrolito en sueros intravenosos. En la industria de los plásticos biodegradables constituye materia prima para sintetizar ácido poli L - láctico (APL). (Araya, 2010; Tejeda et al, 2010; Tenea et al., 2020; Vijayakumar et al., 2008).

La creciente conciencia ambiental en la sociedad ha impulsado las empre- 
sas a emplear plásticos biodegradables como los de APL en empaques y envases. Esta situación incide en la demanda de grandes cantidades de LA. El consumo de este producto fue $1.200 \mathrm{kTon}$ en 2016 , proyectando un crecimiento anual de $16,2 \%$ hasta 2025 , lo que representa aproximadamente 9,8 billones de dólares americanos en el mercado mundial. En la actualidad, el LA posee un precio entre $1,3 \$ / \mathrm{kg}$ a $2,3 \$ / \mathrm{kg}$ y su porcentaje de pureza comercial es del $88 \%$. (Biddy et al., 2016; Oliveira et al., 2018).

Según estas consideraciones, el ácido láctico es un compuesto de interés científico desde hace mucho tiempo; la investigación orientada a su producción abarca el estudio de diferentes sustratos, metodologías de obtención, separación y cuantificación, como también sus potenciales usos. Su obtención a partir del aprovechamiento de residuos agroindustriales permite el acceso a una gran variedad de biomasa lo que brinda muchas alternativas ambientalmente sostenibles, favoreciendo el entorno económico social de los cultivadores de piña, en este caso, especialmente, del departamento Santander.

En 2018, Santander generó $43 \%$ de la producción de piña del país con 455.701
Ton/año. Los municipios de este departamento, con mayor producción fueron Lebrija con 267.250 Ton (59 \%), Rionegro 71.005 Ton (16\%), Girón 47.263 Ton (10\%) y los otros municipios con una producción de 69.469 Ton (15\%). (Gonzales, 2019, Romero, 2019). Teniendo en cuenta que en los cultivos de piña los residuos no aprovechables (hojas, tallos, raíces) generan entre 200 y 250 Ton/ hectárea cosechada, hace que estos sean una biomasa disponible para la obtención de coproductos de interés. (Soto, 2016).

\section{Metodología}

Se realizó una revisión bibliográfica de artículos científicos indexados en la base de datos Scopus (Elsevier, B. V., 2021), con el fin de definir tendencias en investigación, en la obtención del ácido láctico a partir de la piña y sus residuos e identificar los principales microorganismos. Se estructuró la siguiente ecuación de búsqueda (TITLEABS-KEY («lactic acid») AND TITLE-ABS-KEY ( pineapple OR “Ananas comosus" ) ) AND DOCTYPE ( ar ) AND PUBYEAR > 1999. se empleó el programa especializado para minería de texto VantagePoint (Search Technology, Versión académica 12.0). 


\section{Análisis y discusión de resultados}

De acuerdo con la ecuación de búsqueda planteada se obtuvieron 87 artículos indexados en la base de datos Scopus, los cuales referencian estudios para la obtención de ácido láctico a partir de piña. Estos trabajos aplican en diferentes campos de conocimiento (figura 2), como es el caso de la agricultura y ciencias biológicas que presentan 50 documentos relacionados con investigación en alimentos. Un ejemplo de estas publicaciones - desarrollada por Nguyen et al., (2019, p. 1) — estudió la fermentación del jugo de piña utilizando cepas de bacterias de ácido láctico (Lactobacillus y Bifidobacterium) donde se halló cambios en la estabilidad de las bebidas durante el almacenamiento.
En los campos de bioquímica, genética y biología molecular y ciencia de los materiales se encontraron 19 y 17 artículos registrados, respectivamente. Las aplicaciones en estos campos están relacionadas con biotecnología, microbiología, farmacia y producción de biopolímeros. El siguiente campo de conocimiento con mayor relevancia en cuanto a cantidad de artículos publicados es química con 16 artículos; en la investigación de (Díaz-Vela et al., 2015, p. 2636) se evaluó el potencial que posee la cáscara de frutas como nopal y piña - ingredientes funcionales, fuente de fibra, textura y probióticos- en productos cárnicos cocidos. Uno de los resultados relevantes fue la mejora en el desarrollo de las bacterias de ácido láctico en los productos cárnicos durante su almacenamiento.

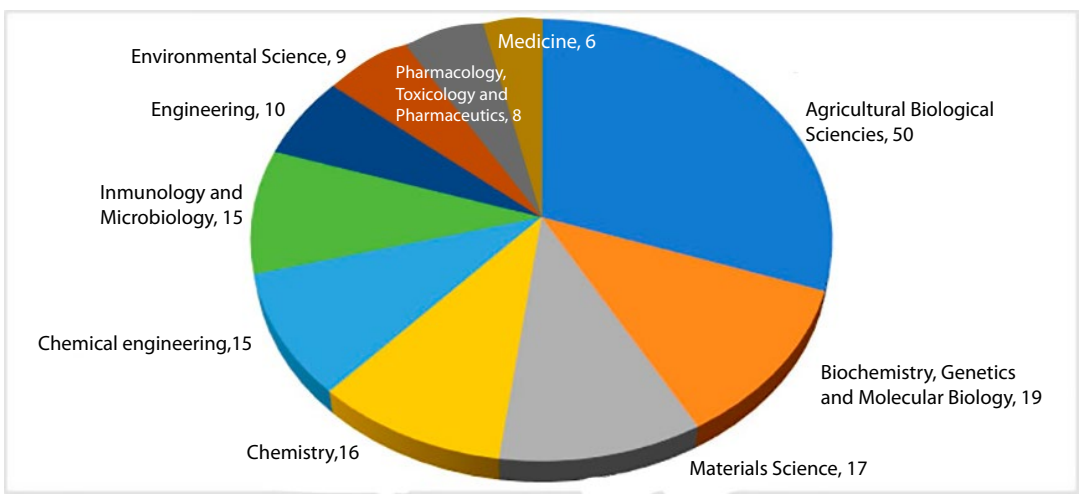

Figura 2. Distribución del interés de la obtención de ácido láctico a partir de la piña. Nota. Fuente: Unidad de Bibliometría- CRAI Biblioteca Universidad Santo Tomás, Seccional Bucaramanga. Cálculos basados en información de la base de datos Elsevier (Scopus B.V., 2021), procesados con VantagePoint (Search Technology, versión académica 12.0). 
En la figura 3 se presenta la dinámica científica, la cual se mide por número de artículos publicados por año. En este caso, se definió el período de revisión entre 2010 y 2020. En 2012 solo se registró una publicación relacionada con el uso de técnicas instrumentales para la caracterización de compuestos volátiles en panes de piña (Ying et al., 2012, p. 13802). Para 2015 la cantidad de registros aumentó a 12, observándose una tendencia en el estudio de esta fruta en el campo de la agricultura y ciencias biológicas. Finalmente, el año de mayor actividad fue 2020 con 15 publicaciones. Estos trabajos aplican en diferentes campos del conocimiento donde predomina el campo investigativo de inmunología y microbiología seguido de ciencia de materiales y, en una menor proporción, agricultura y ciencias biológicas.
En cuanto a distribución por países (figura 4) destaca Tailandia con 15 publicaciones relacionadas con aprovechamiento de desechos de piña en agricultura y ciencias biológicas, ingeniería química y ciencia de los materiales debido, quizás, a que este país se encuentra entre los principales a nivel mundial - ocupa el puesto número cuatro- en producción de esta fruta, según la FAO. (Food and Agriculture Organization (FAO), 2017). Por otro lado, Malasia, que ocupa la posición 18 , también presenta 15 publicaciones -quizá porque este país clasificaba como uno de los principales productores de piña a nivel mundial hasta 2000 y aunque su producción ha disminuido sigue haciendo parte de los 20 mayores productores en la actualidad (Pariona, 2018)-.

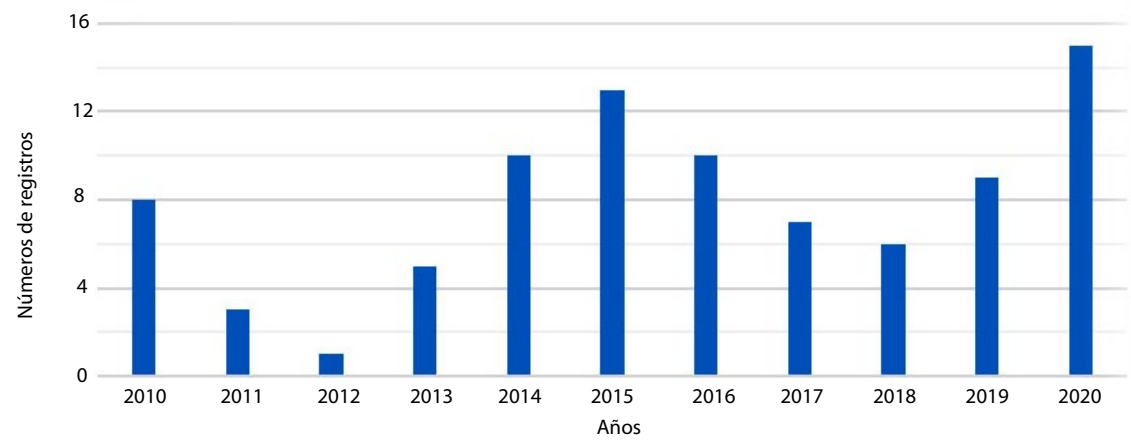

Figura 3. Dinámica científica obtención de ácido láctico a partir de la piña.

Nota. Fuente: Unidad de Bibliometría- CRAI Biblioteca Universidad Santo Tomás, Seccional

Bucaramanga. Cálculos basados en información de la base de datos Elsevier (Scopus B.V., 2021), procesados con VantagePoint (Search Technology, versión académica 12.0). 
A nivel de Latinoamérica sobresalen México y Brasil con siete y cuatro publicaciones, respectivamente. México es considerado un productor pequeño de piña a nivel mundial, sin embargo, es el segundo mayor proveedor de esta fruta a Estados Unidos de América (Food and Agriculture Organization (FAO), 2019). Las publicaciones se relacionan con procesos biotecnológicos de fermentación de diferentes partes de la fruta. Por su parte, los trabajos de Brasil se relacionan con el aprovechamiento de residuos de la piña en el campo de los biopolímeros y alimentos; este país ocupa el tercer puesto a nivel mundial en cuanto a la producción de piña, la cual es destinada principalmente para el consumo interno (Food and Agriculture Organization (FAO), 2018)

Colombia solo aparece con un artículo indexado en la base de datos, de acuerdo con la ecuación de búsqueda empleada, donde emplearon desechos de piña variedad oro miel y subproductos de Sacha inchi; caracterizaron su forma elemental, física y química con el fin de formular un sustrato de fermentación suplementado (SFS) para el crecimiento de Weissella cibaria y hubo una producción de biomasa de 2,93 g/L con viabilidad de 9,88 $\log \mathrm{CFU} / \mathrm{mL}$ (Micanquer-Carlosama et al., 2020).

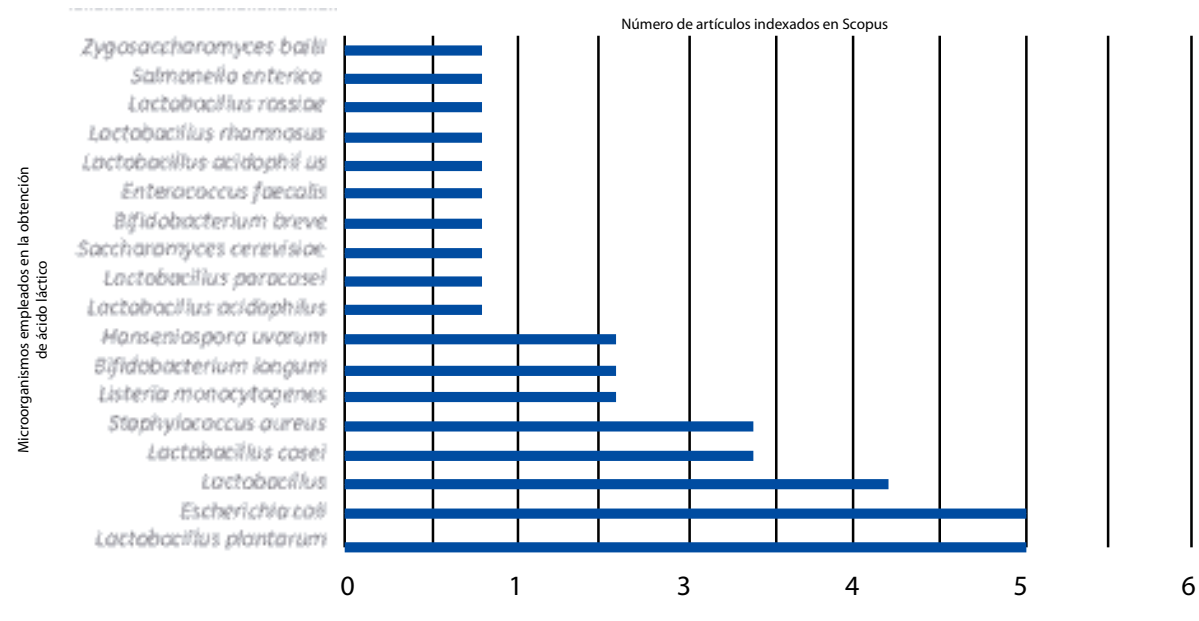

Figura 4. Distribución por países de artículos relacionados con la obtención de ácido láctico a partir de la piña.

Nota. Fuente: Unidad de Bibliometría- CRAl Biblioteca Universidad Santo Tomás, Seccional Bucaramanga. Cálculos basados en información de la base de datos Elsevier (Scopus B.V., 2021), procesados con VantagePoint (Search Technology, versión académica 12.0). 
Se realizó también el análisis de co-ocurrencia de las palabras clave, el cual se incluye dentro de los indicadores relacionales que permiten identificar tendencias en la temática de interés, en este caso, el estudio del ácido láctico. Para visualizar la relación entre términos empleados por los autores se empleó el programa gratuito Vosviewer (2021, Centre for Science and Technology Studies, Leiden University, The Netherlands, versión 1.6.15).
En la figura 5 se observa la relación que presentan las palabras clave asociadas con la obtención del ácido láctico a partir de la piña y sus residuos. Un grupo grande de estas palabras describen procesos fermentativos y biotecnológicos, en los cuales se emplean microorganismos tipo probióticos. Otro clúster de palabras está asociado con la aplicación del ácido poliláctico como polímero biodegradable, el uso de las fibras como refuerzos estructurales de otros biopolímeros, entre otros.

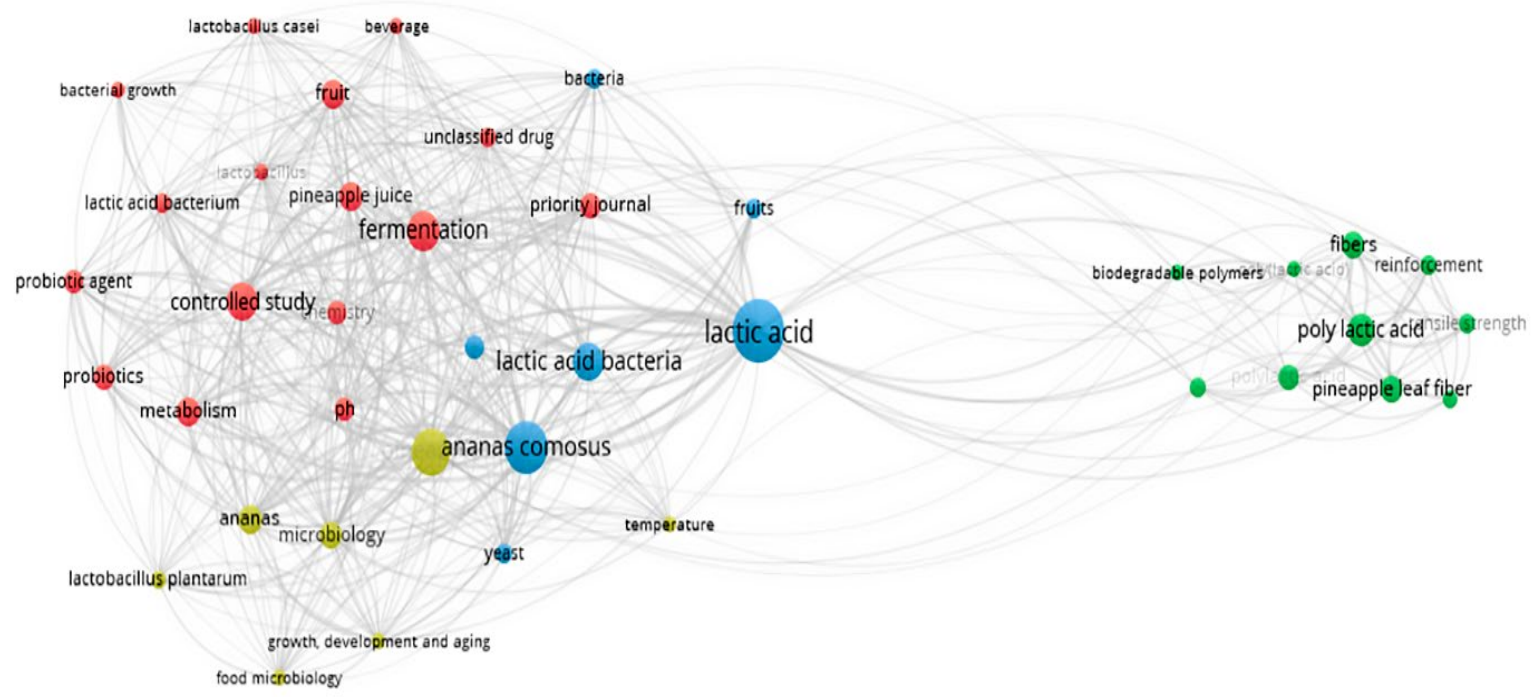

Figura 5. Relación de las palabras clave de mayor co-ocurrencia.

Nota. Fuente: Unidad de Bibliometría del CRAI Biblioteca de la Universidad Santo Tomás, Seccional Bucaramanga, cálculos basados en información de Scopus (Elsevier, 2021) y analizados con el programa gratuito VOSviewer versión 1.6.15 
Se analizaron términos asociados con piña y microorganismos para encontrar términos asociados con piña y microorganismos empleados para la obtención de ácido láctico — se identificaron 18; la gran mayoría pertenece al género Lactobacillus, destacando el Lactobacillus plantarum (5), seguido por el microorganismo Escherichia coli (5). Algunos autores tan solo definieron el género Lactobacillus (4), como se observa en la figura 6-. Entre otros microorganismos que han sido evalua- dos para la obtención de ácido láctico se encuentran Staphylococcus aureus (3), Listeria monocytogenes (2), Bifidobacterium longum (2), Hanseniaspora uvarum (2), Saccharomyces cerevisiae (1), Bifidobacterium breve (1), Enterococcus faecalis (1), Salmonella enterica (1) y Zygosaccharomyces bailii (1). Los trabajos en los cuales se emplea Escherichia coli han sido reportados por investigadores de Malasia, Indonesia e Italia. El Staphylococcus aureus se estudió en Malasia e Indonesia.

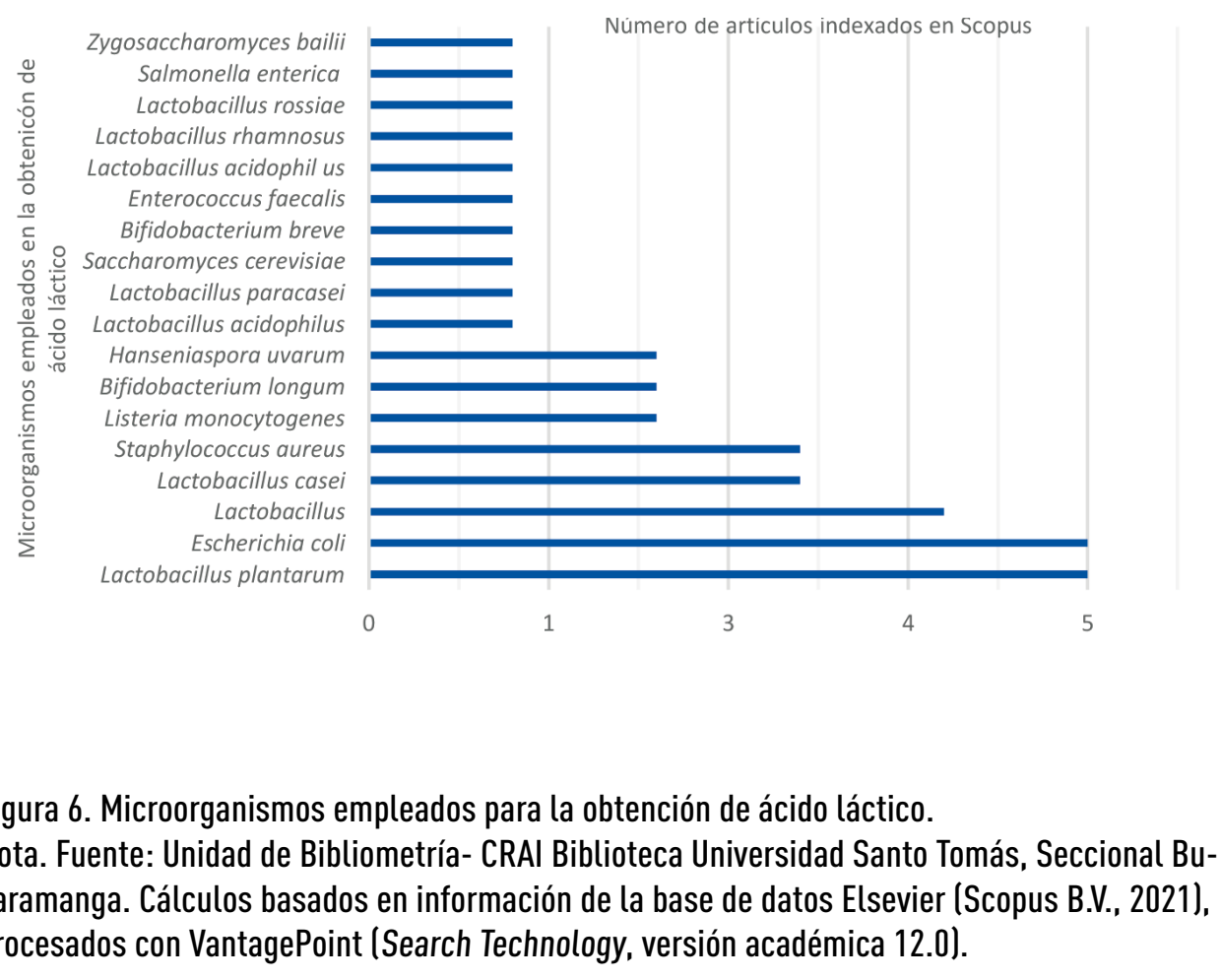


Para la obtención de ácido láctico los autores reportaron el uso de la fruta de piña (18), también las hojas de la planta (10), el jugo de esta fruta (10) y los residuos de piña (4), como se aprecia en la figura 7. La obtención de LA a partir de desechos de piña llevó al avance de investigaciones utilizando diferentes partes de la fruta. Un ejemplo de esto es la investigación desarrollada por (Escobar-Ramírez et al., 2020) donde se empleó la bebida fermentada de la piña para aislar bacterias lácticas con potencial probiótico. Igualmente, los desechos de cáscara de piña fueron empleados en el estudio de (Ngouénam et al., 2021) para obtener ácido láctico utilizando cepas de Lactobacillus sp aislada de residuos de frutas tropicales (plátano, papaya, piña y naranja), mediante fermentación y se obtuvo una concentración máxima de ácido láctico a partir de la piña de $26,29 \mathrm{~g} / \mathrm{L}$. En otra investigación, se evaluó el potencial de un desecho agroindustrial de piña para la producción de ácido láctico por fermentación, utilizando Lactobacillus casei subespecie rhamnosus, en esta investigación se obtuvo una concentración máxima de ácido láctico de $75 \mathrm{~g} / \mathrm{L}$, debido al consumo total de los azúcares presentes en el medio hidrolizado (Araya et al., 2010).

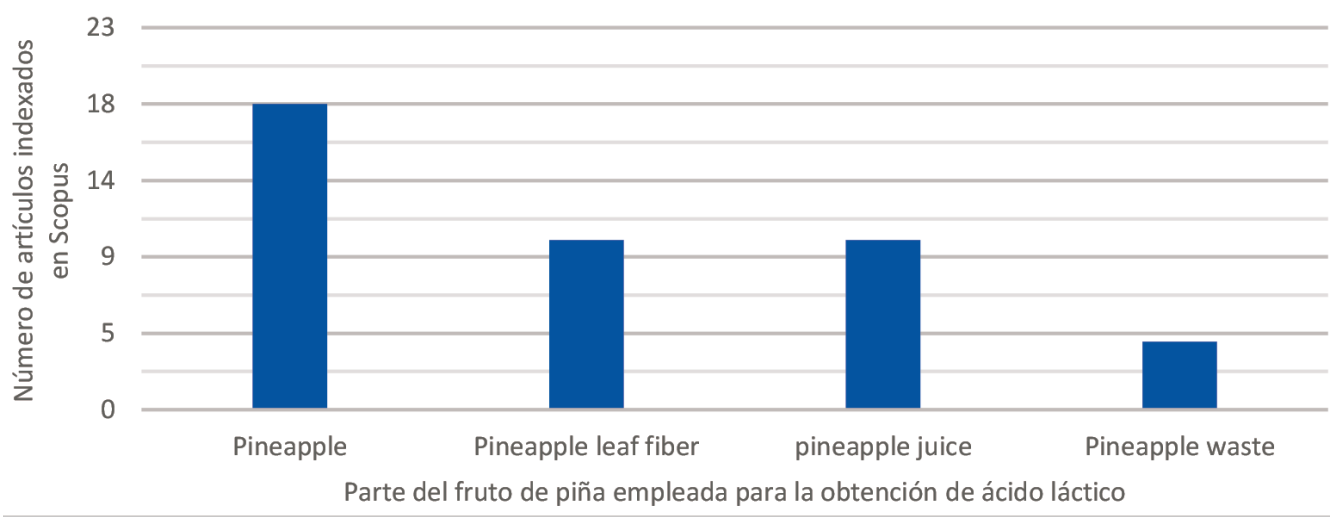

Figura 7. Partes de la fruta de piña empleadas para la obtención de ácido láctico. Nota. Fuente: Unidad de Bibliometría- CRAI Biblioteca Universidad Santo Tomás, Seccional Bucaramanga. Cálculos basados en información de la base de datos Elsevier (Scopus B.V., 2021), procesados con VantagePoint (Search Technology, versión académica 12.0). 


\section{Conclusiones}

El interés por el estudio del aprovechamiento de la piña y sus residuos para la obtención no tiene una tendencia definida, sin embargo, se puede apreciar un aumento en el número de publicaciones asociadas con este tema, especialmente en Malasia y Tailandia, países catalogados también como los mayores productores de la fruta. Se identificó también que los investigadores orientan los métodos de obtención aplicando procesos biotecnológicos en los cuales introducen diversos microorganismos buscando condiciones óptimas de producción. Considerando lo anterior, se identificaron cerca de 18 microorganismos que han sido evaluados para la obtención de ácido láctico a partir de residuos de piña. Los más empleados corresponden al género Lactobacillus. Sin embargo, también se encontraron microorganismos como la Escherichia coli, la cual en el campo biotecnológico es una enterobacteria útil por su velocidad de crecimiento $\mathrm{y}$ pocas necesidades nutricionales, entre otras ventajas que presenta. Este tipo de investigaciones buscan no solo el desarrollo de tecnologías de aprovechamiento, sino que sean ambientalmente amigables, de manera que se reduzca el impacto negativo ocasionado por la disposición final de este tipo de residuos biodegradables. También se considera el impacto socio económico para los productores de piña, pues estos procesos de valorización permitirían generar nuevos ingresos para este sector.

\section{Agradecimientos}

Los autores agradecen a la Unidad de Bibliometría del CRAI, Biblioteca Universidad Santo Tomás, seccional Bucaramanga.

\section{Referencias}

Aida, H. I., Mahanom, H., \& Norhartini, A. S. (2011). Dietary fibre powder from pineapple by-product as a potential functional food ingredient. Acta Horticulturae, 902, 565-568. https://doi.org/10.17660/actahortic. 2011.902 .75

Araya-Cloutier, C., Rojas-Garbanzo, C., \& Velázquez-Carillo, C. (2010). Síntesis de ácido láctico, a través de la hidrólisis enzimática simultánea a la fermentación de un medio a base de un desecho de piña (Ananas comosus), para su uso como materia prima en la elaboración de ácido poliláctico. Revista Iberoamericana de Polímeros, 11(7), 407-416.

Beltrán-Ramírez, F., Orona-Tamayo, D., Cornejo-Corona, I., Luz Nicacio González-Cervantes, J., de Jesús Esparza-Claudio, J., y Quintana-Rodríguez, E. (2019). Agro-Industrial Waste Revalorization: The Growing Biorefinery. In Biomass for Bioenergy - Recent Trends and Future Challenges. IntechOpen. https://doi.org/10.5772/intechopen.83569 
Biddy, Mary J., Scarlata, Christopher, \& Kinchin, Christopher. (2016). Chemicals from Biomass: A Market Assessment of Bioproducts with Near-Term Potential. United States. https://doi.org/10.2172/1244312

Díaz-Vela, J., Totosaus, A., \& Pérez-Chabela, M. L. (2015). Integration of Agroindustrial Co-Products as Functional Food Ingredients: Cactus Pear (Opuntia ficus indica) Flour and Pineapple (Ananas comosus) Peel Flour as Fiber Source in Cooked Sausages Inoculated with Lactic Acid Bacteria. Journal of Food Processing and Preservation, 39(6), 2630-2638. https://doi. org/10.1111/jfpp. 12513

Escobar-Ramírez, M. C., Jaimez-Ordaz, J., Escorza-Iglesias, V. A., Rodríguez-Serrano, G. M., Contreras-López, E., Ramírez-Godínez, J., Castañeda-Ovando, A., Morales-Estrada, A. I., Felix-Reyes, N., \& González-Olivares, L. G. (2020). Lactobacillus pentosus ABHEAU-05: An in vitro digestion resistant lactic acid bacterium isolated from a traditional fermented Mexican beverage. Revista Argentina de microbiología, 52(4), 305-314. https://doi. org/10.1016/j.ram.2019.10.005

Food and Agriculture Organization (FAO). (2017). Perspectivas mundiales de las principales frutas tropicales. http://www. fao.org/fileadmin/templates/est/COMM MARKETS_MONITORING/Tropical_ Fruits/Documents/Tropical_Fruits_Spanish2017.pdf

Food and Agriculture Organization (FAO). (2018). Las principales frutas tropicales. Análisis de mercado. http://www.fao.org/3/ ca5692es/CA5692ES.pdf
Food and Agriculture Organization (FAO). (2019). Análisis del mercado de las principales frutas tropicales. http://www.fao. org/3/cb0834es/cb0834es.pdf

Gonzales, X. (14 de agosto de 2019). Agronegocios. Obtenido de https://www. agronegocios.co/agricultura/la-produccion-de-pina-en-colombia-llegaria-a118-millones-de-toneladas-al-finalizar-el-ano-2895397

Ketnawa, S., Chaiwut, P. y Rawdkuen, S. (2012). Residuos de piña: una fuente potencial de extracción de bromelina. Procesamiento de alimentos y bioproductos, 90, 385-391. doi: 10.1016 / j.fbp.2011.12.006

Kuan, Y. H., \& Liong, M. T. (2008). Chemical and physicochemical characterization of agrowaste fibrous materials and residues. Journal of agricultural and food chemistry, 56(19), 9252-9257. https://doi. org/10.1021/jf802011j

Micanquer-Carlosama, A., Cortés-Rodríguez, M., \& Serna-Cock, L. (2020). Formulation of a fermentation substrate from pineapple and sacha inchi wastes to grow Weissella cibaria. Heliyon, 6(4), e03790. https://doi. org/10.1016/j.heliyon.2020.e03790

Ngowénam, J., Kenfack, C., Kouam, M., Kaktchman, P., Maharjan, R., Ngoufack, F. (2021) Lactic acid production ability of Lactobacillus sp. From four tropical fruits using their by-products as carbon source. Heliyon, 7 (5). https://doi.org/10.1016/j. heliyon.2021.e07079

Nguyen, B. T., Bujna, E., Fekete, N., Tran, A. T. M., Rezessy-Szabo, J. M., Prasad, R., \& Nguyen, Q. D. (2019). Probiotic Beverage From Pineapple Juice Fermented With 
Lactobacillus and Bifidobacterium Strains. Frontiers in Nutrition, 6, 1. https://doi. org/10.3389/fnut.2019.00054

Oliveira, R., Komesu, A., Rossell, C.E., \& Filho, R.M. (2018). Challenges and opportunities in lactic acid bioprocess design-From economic to production aspects. Biochemical Engineering Journal, 133, 219-239.

Pariona, A. (2018, 19 abril). Top Pineapple Producing Countries. WorldAtlas. https:// www.worldatlas.com/articles/top-pineapple-producing-countries.html

Romero, M. (2019). Plan de negocios de exportación de piña hacia Estados Unidos. Bogotá: Fundación Universitaria de América.

Salcedo, J. M. (2016). Piña (Ananas sp) [Fotografia]. Repositorio Institucional de Documentación Científica Instituto de Investigación de Recursos Biológicos Alexander von Humboldt. http://repository.humboldt.org. co/handle/20.500.11761/1520

Vargas, A. (2016). Estudio técnico para la valorización de residuos sólidos orgánicos vegetales provenientes de la Plaza de Mercado San Francisco de Bucaramanga. Bucaramanga: Universidad Santo Tomás, seccional Bucaramanga.
Torre Pascual, I. (2019). Producción biotecnológica de ácido D-Láctico a partir de residuos de naranja. Universidad Complutense de Madrid, 1 - 219.

Tenea, G. N., Olmedo, D., \& Ortega, C. (2020). Peptide-Based Formulation from Lactic Acid Bacteria Impairs the Pathogen Growth in Ananas Comosus (Pineapple). Coatings, 10(5), 457. https://doi.org/10.3390/ coatings 10050457

Vijayakumar, J., Aravindan, R. y Viruthagiri, T. (2008). Recent trends in the production, purification and application of lactic acid. Chemical and Biochemical Engineering Quarterly, 22(2), 245-264

Ying, S., Lasekan, O., Naidu, K., \& Lasekan, S. (2012). Headspace Solid-Phase Microextraction Gas Chromatography-Mass Spectrometry and Gas Chromatography-Olfactometry Analysis of Volatile Compounds in Pineapple Breads. Molecules, 17(12), 13795-13812. https://doi.org/10.3390/molecules 171213795 